\title{
APTITUD COMBINATORIA GENERAL Y ESPECÍFICA EN LA CALIDAD DE SEMILLA DE LÍNEAS S ${ }_{3}$ DE MAÍZ
}

\author{
GENERAL AND SPECIFIC COMBINING ABILITY ON SEED QUALITY OF $S_{3}$ MAIZE INBRED LINES
}

\author{
Francisco Cervantes-Ortiz * , Janianny Hernández-Esparza', J. Antonio Rangel-Lucio², \\ Enrique Andrio-Enríquez ${ }^{1}$, Mariano Mendoza-Elos ${ }^{1}$, Gilberto Rodríguez-Pérez ${ }^{1}$ \\ y Luis P. Guevara-Acevedo'
}

\begin{abstract}
${ }^{1}$ Instituto Tecnológico de Roque. Km. 8 Carretera Celaya-J. Rosas, sin número. 38110, Celaya, Guanajuato, México. ${ }^{2}$ Instituto Tecnológico de Victoria. Boulevard Emilio Portes Gil \# 1301 Poniente. 87010, Ciudad Victoria, Tamaulipas, México.
\end{abstract}

*Autor de correspondencia (frcervantes@itroque.edu.mx)

\section{RESUMEN}

El análisis de cruzas dialélicas es una herramienta útil para determinar el efecto genético en estudios de calidad de semilla de maíz (Zea mays L.). El objetivo de esta investigación fue determinar los componentes genéticos de aptitud combinatoria general (ACG), aptitud combinatoria específica (ACE) y efectos recíprocos (ER) involucrados en la expresión de la calidad de semilla y vigor de plántula de líneas $\mathrm{S}_{3}$ y sus cruzas directas y recíprocas. Las cruzas $F_{1}$ y sus progenitores se sometieron a pruebas analíticas de calidad física como biomasa de semilla y calidad fisiológica mediante germinación estándar, velocidad de germinación y vigor por envejecimiento acelerado. Adicionalmente, se desarrolló un ensayo en arena como sustrato para registrar la emergencia y altura de plántulas, biomasa seca del vástago, índice de vigor I e índice de vigor II. Se utilizó un diseño experimental de bloques completos al azar con tres repeticiones en laboratorio y con dos repeticiones en la evaluación en cama de arena. Se encontraron diferencias estadísticas significativas entre genotipos en la calidad de la semilla y el vigor de la plántula. Situación semejante ocurrió para los efectos de ACG, ACE y ER en ambos grupos de características evaluadas, con predominio de ACG sobre ACE. Los efectos recíprocos significativos indican la importancia de seleccionar apropiadamente el progenitor femenino para favorecer la calidad de la semilla y el vigor de la plántula. En las cruzas con heterosis alta en estos rasgos participó cuando menos una línea de alta ACG y los efectos de ACE fueron altos y positivos. Las líneas de maíz TNM- 5 y TNM-4 mostraron alta ACG y alta ACE en combinaciones específicas superiores, por lo que podrían ser empleadas primero en programas de mejoramiento genético por selección para incrementar la frecuencia genética aditiva en la calidad de semilla y vigor de plántula y posteriormente usarse en programas de hibridación. ACE.

Palabras clave: Zea mays, cruzas dialélicas, efectos genéticos, ACG,

\section{SUMMARY}

Analysis of diallel crosses is an important tool to determine the genetic effect in studies of seed quality of maize (Zea mays L.). The objective of this research was to determine the genetic component of general combining ability (GCA), specific combining ability (SCA) and reciprocal effects (ER) involved in the expression of seed quality and seedling vigor of $\mathrm{S} 3$ lines, as well as of their direct and reciprocal crosses. The $F_{1}$ crosses and their parents were submitted to analytical tests of physical quality as seed biomass and seed physiological quality by standard germination, germination speed and vigor by accelerated aging. Additionally, an assay was developed in sand as a substrate for recording in seedling emergence, seedling height, shot dry biomass, vigor index I and II. A complete randomized blocks design with three replications was used in laboratory and with two replications in the evaluation in the sand bed. Statistically significant differences between genotypes were found in seed quality and seedling vigor. A similar situation occurred for GCA, SCA and RE in both groups of the evaluated traits, where ACG was predominant over SCA. Significant reciprocal effects showed the importance of properly selecting the female parent to promote the seed quality and seedling vigor The crosses with high heterosis for these characters had at least one high GCA inbred line, and SCA effects were high and positive. Maize lines TNM-5 and TNM-4 showed high GCA and high SCA in specific superior combinations; thus they might be used in breeding programs: first for selection to increase the additive genetic frequency on seed quality and seedling vigor, and later they might be used in hybridization programs.

Index words: Zea mays, diallel crosses, genetic effects, GCA, SCA.

\section{INTRODUCCIÓN}

Los análisis dialélicos constituyen una herramienta útil para caracterizar y estimar los parámetros genéticos de progenitores y sus cruzas, mediante su aptitud combinatoria general (ACG) y aptitud combinatoria específica (ACE); además, permiten definir el método más adecuado de mejoramiento genético para predecir cruzas superiores y combinar las mejores características de los progenitores (Hallauer y Miranda, 1981). Entre los métodos existentes para evaluar las cualidades de un conjunto de progenitores se encuentran los diseños dialélicos propuestos por Griffing (1956), porque permiten identificar los mejores progenitores y las mejores combinaciones entre ellos. Resultados diversos confirman la importancia de los diseños dialélicos utilizados en mejoramiento genético de plantas para estimar ACG y ACE de los progenitores y clasificarlos en grupos heteróticos (Cai et al., 2012; Melani y Carena, 2005; Yan y Kang, 2003).

Existen dos hipótesis principales que explican el fenómeno de heterosis: la de dominancia y la de sobre-dominancia (Allard, 1960). De acuerdo con Crow (1999) en términos de acción génica, la heterosis se debe principalmente a efectos de interacción entre alelos o dominancia. En forma operativa, la heterosis se calcula como la diferencia entre el valor fenotípico de la $F_{1}$ y el valor del progenitor medio o el del progenitor superior y se expresa en porcentaje (Falconer y Mackay, 1996). 
La calidad de la semilla está definida por la suma de atributos genéticos, físicos, fisiológicos y sanitarios, los cuales determinan su germinación, vigor y longevidad (Goggi et al., 2008; Popinigis, 1985). El uso de semillas con alta calidad favorece la mayor productividad en campo (FinchSavage, 1994; Munamava et al., 2004), debido a que un establecimiento deficiente del cultivo en campo se traduce en rendimiento bajo en la mayoría de las especies cultivadas (Marcos-Filho, 2005). Por tanto, es importante investigar el efecto genético y su relación con la calidad de la semilla (TeKrony y Egli, 1991).

A pesar de que el maíz (Zea mays L.) es un cultivo ampliamente estudiado, existe poco interés por involucrar los efectos genéticos en la calidad de la semilla y vigor inicial de plántula (Moterle et al., 2011; Silva et al., 2013). En este sentido, Vergara et al. (2005) recomiendan conocer el comportamiento genético de las características de importancia económica de cada población de maíz, con el propósito de elegir la estrategia de selección y obtener híbridos con mayores ventajas agronómicas.

Hoecker et al. (2006) estimaron una heterosis de hasta 51 \% en plántulas de líneas de maíz de grano cristalino y dentado, mientras que Cervantes-Ortiz et al. (2007) determinaron que la varianza de dominancia era más importante en el vigor inicial de plántulas de maíz. En contraste, en líneas de maíz de Valles Altos Barla-Szabo et al. (1989) encontraron que los efectos aditivos son los más importantes en el vigor de la semilla. Otros estudios en el campo genético (Cervantes et al., 2006; Moterle et al., 2011) revelaron mayores efectos de genes aditivos que de dominancia en la calidad de semilla en maíz.

Por otro lado, Antuna et al. (2003) y Silva et al. (2013) encontraron en maíz valores superiores de ACE para germinación estándar, velocidad de germinación, vigor de semilla y vigor inicial de plántula, lo que indica la predominancia de una acción génica no aditiva. Otro estudio que involucró el peso de la semilla con metas similares, reveló efectos significativos para ACG, ACE y efectos recíprocos $(E R)$, además de una correlación positiva con otras carac- terísticas asociadas con la calidad de semilla (Revilla et al., 1999). En este contexto, Gomes et al. (2000) y Moterle et al. (2012) encontraron que la varianza de la ACE fue mayor que la varianza de la ACG, lo que revela mayor importancia de los efectos de dominancia que los efectos aditivos en la calidad de semilla.

Con base en lo anterior, el objetivo de este estudio fue determinar los componentes genéticos de ACG, ACE y ER involucrados en la expresión de la calidad de semilla y vigor de plántula de líneas $\mathrm{S}_{3}$ de maíz y en sus cruzas directas y recíprocas. La hipótesis del estudio fue que la calidad de la semilla y vigor inicial de las plántulas de maíz están determinados principalmente por efectos de genes aditivos.

\section{MATERIALES Y MÉTODOS}

Cinco líneas de maíces blancos con grado de endogamia $\mathrm{S}_{3}$ del programa de mejoramiento genético del Instituto Tecnológico de Roque (ITR; Cuadro 1), fueron cruzadas en forma directa y recíproca para generar un dialélico completo de 20 combinaciones bajo el método I de Griffing (1956).

Las cruzas se formaron en el ciclo de Primavera-Verano 2013 en el campo experimental del ITR y al mismo tiempo se incrementaron las líneas, para obtener un total de 25 genotipos: 10 cruzas directas, 10 cruzas recíprocas y cinco progenitores, los cuales fueron cosechados por separado en octubre de 2013.

Con una muestra de semillas de las cruzas $F_{1}$ directas y recíprocas y de sus progenitores se hicieron pruebas analíticas para determinar calidad física y fisiológica de semilla en el Laboratorio de Análisis de Calidad de Semilla del ITR; donde se registró biomasa de semilla (BS), germinación estándar (GE), velocidad de germinación en el primer recuento (VG) y vigor de semilla por envejecimiento acelerado (EA), mediante el uso de un diseño de bloques completos al azar con tres repeticiones. El tamaño de muestra para BS fue de 100 semillas y para GE, VG y EA de 50 semillas. Las pruebas se realizaron aplicando la metodología de la International Seed Testing Association (ISTA, 1995).

Cuadro 1. Origen y características agronómicas de las líneas de maíz $\mathrm{S}_{3}$ empleadas en el diseño de cruzas dialélicas.

\begin{tabular}{lccccc}
\hline No. & Línea & Nivel de endogamia & Origen de las líneas & Origen del híbrido & Color de grano \\
\hline 1 & TNM-1 & $\mathrm{S}_{3}$ & Pob. $F_{2}$ (DK2042) & Monsanto & Blanco \\
2 & TNM-2 & $\mathrm{S}_{3}$ & Pob. $F_{2}$ (DK2027) & Monsanto & Blanco \\
3 & TNM-3 & $\mathrm{S}_{3}$ & Pob. $F_{2}($ P3030W) & Pioneer & Blanco \\
4 & TNM-4 & $\mathrm{S}_{3}$ & Pob. $F_{2}$ (Caimán) & Monsanto & Blanco \\
5 & TNM-5 & $\mathrm{S}_{3}$ & Variedad Roque & ITR & Blanco \\
\hline
\end{tabular}

Pob = población; ITR = Instituto Tecnológico de Roque. 
Los mismos genotipos se sembraron en invernadero en camas de arena, en febrero de 2014, para evaluar vigor inicial de plántula. El diseño experimental utilizado fue bloques completos al azar con dos repeticiones. El tamaño de la unidad experimental fue de 25 semillas por genotipo y las variables medidas fueron: porcentaje de emergencia $(\mathrm{PE})$, altura de plántula (APL), biomasa seca de vástago (BSV), índice de vigor I (IV-I) e índice de vigor II (IV-II) de plántulas; estos índices se calcularon como el producto del porcentaje de emergencia por la longitud de plántula y del porcentaje de emergencia por la biomasa seca de vástago, respectivamente. El porcentaje de emergencia se obtuvo por el recuento de plántulas emergidas al séptimo día después de la siembra. La altura de plántula y la biomasa seca de vástago se midieron 15 d después de la siembra en una muestra de diez plantas con competencia completa por unidad experimental.

Para cada variable se realizó un análisis de varianza para determinar la aptitud combinatoria general (ACG), específica (ACE) y efectos recíprocos (ER), de acuerdo con el Método I de Griffing (1956); para ello se usó el programa de cómputo Diallel-SAS Method I (Zhang y Kang, 2003).

\section{RESULTADOS Y DISCUSIÓN}

Los genotipos mostraron diferencias estadísticas significativas $(P \leq 0.01)$ para las características de calidad de semilla (Cuadro 2), como biomasa de semilla (PS), velocidad de germinación (VG) registrada al cuarto día, germinación estándar (GE) y vigor de la semilla evaluado con la prueba de envejecimiento acelerado (EA) seguida por la prueba de germinación estándar. Con base en la descomposición de los cuadrados medios de los genotipos, se encontró que ACG, ACE y ER presentaron efectos estadísticos significativos $(P \leq 0.01)$ en este grupo de variables.

En vigor de plántula, determinado por la emergencia (E), altura de plántula (APL), índice de vigor I (IV-I) e índice de vigor II (IV-II), también se observaron diferencias estadísticas altamente significativas entre genotipos $(P \leq 0.01)$. Del mismo modo, en la descomposición de los cuadrados medios para los efectos de ACG, ACE y ER se presentaron diferencias estadísticas altamente significativas $(P \leq 0.01)$ en las características (Cuadro 3); sin embargo, en biomasa seca de vástago (BSV) sólo hubo efecto estadístico significativo $(P \leq 0.01)$ para ACG.

Cuadro 2. Cuadrados medios del análisis dialélico en características de calidad física y fisiológica de semilla en genotipos de maíz.

\begin{tabular}{|c|c|c|c|c|c|}
\hline $\mathrm{FV}$ & $\mathrm{GL}$ & $\mathrm{BS}(\mathrm{g})$ & VG (\%) & GE (\%) & EA (\%) \\
\hline Repeticiones & 2 & 2.5 & 85.1 & 17.9 & 27.5 \\
\hline Genotipos & 24 & $65.3 * \star$ & $1056.1 * *$ & $438.2 * \star$ & $1176.5 * \star$ \\
\hline ACG & 4 & $209.2 * *$ & $1312.4 * *$ & $739.8 * *$ & $2497.1 * *$ \\
\hline ACE & 10 & $47.7 \star \star$ & $1807.9 * \star$ & $256.7 \star \star$ & $1484.0 * \star$ \\
\hline ER & 10 & $25.4 * *$ & 201.9* & $499.0 * *$ & $340.8 * *$ \\
\hline Error & 48 & 1.5 & 84.4 & 9.9 & 34.1 \\
\hline CV (\%) & - & 3.4 & 13.6 & 3.3 & 6.7 \\
\hline
\end{tabular}

*, ** Diferente de cero a una probabilidad de 0.05 y 0.01, respectivamente. FV: fuente de variación; GL: grados de libertad; CV: coeficiente de variación; BS: biomasa de semilla; VG: velocidad de germinación ( $4^{\circ}$ día); GE: germinación estándar; EA: envejecimiento acelerado.

Cuadro 3. Cuadrados medios del análisis dialélico para características de vigor inicial de plántula en genotipos de maíz.

\begin{tabular}{lcccccc}
\hline FV & $\mathrm{GL}$ & $\mathrm{E}(\%)$ & $\mathrm{APL}(\mathrm{cm})$ & $\mathrm{BSV}(\mathrm{g})$ & $\mathrm{IV}-\mathrm{I}$ & $\mathrm{IV}-\mathrm{II}$ \\
\hline Repeticiones & 1 & 32.0 & 0.05 & 0.06 & 226,814 & 883 \\
Genotipos & 24 & $222.3^{* *}$ & $6.38^{* *}$ & 0.17 & $40,419 * *$ & $4812^{* *}$ \\
ACG & 4 & $671.0^{* *}$ & $19.74 * *$ & $0.41 * *$ & $119,709^{* *}$ & $14,292^{* *}$ \\
ACE & 10 & $199.2^{* *}$ & $5.96 * *$ & 0.13 & $36,900^{* *}$ & $3786^{* *}$ \\
ER & 10 & $65.9 * *$ & $1.45^{* *}$ & 0.13 & $12,223^{* *}$ & $2045^{* *}$ \\
Error & 24 & 13.3 & 0.11 & 0.02 & 2205 & 175 \\
CV $(\%)$ & - & 4.2 & 3.15 & 21.15 & 15.5 & 8.5 \\
\hline
\end{tabular}

**Diferente de cero a una probabilidad de 0.01. FV: fuente de variación; GL: grados de libertad; CV: coeficiente de variación; E: emergencia; APL: altura de plántula; BSV: biomasa seca de vástago; IV-I: índice de vigor I; IV-II: índice de vigor II. 
La significancia estadística de los cuadrados medios reveló la existencia de variabilidad genética resultante tanto de efectos aditivos como de dominancia en el control de la expresión génica. En este respecto, las líneas TNM-1, TNM-2 y TNM-4 se derivaron de las poblaciones híbridas $F_{2}$ de la empresa Monsanto y se infiere que tienen algún parentesco entre ellas; en cambio, las líneas TNM-3 y TNM-5 se obtuvieron de una población híbrida $F_{2}$ de la empresa Pioneer y de una variedad de polinización libre, y por ende deben ser diferentes en su composición genética. Según Guillen-De la Cruz et al. (2009) a medida que se incrementa la diversidad genética de los progenitores también se incrementan las diferencias entre sus cruzas, tanto en características agronómicas como fisiológicas.

La significancia estadística de ACG y ACE (Cuadros 2 y 3) indica la importancia de los efectos genéticos aditivos y dominantes involucrados en la calidad de semilla y vigor de plántula, como lo reportaron Antuna et al. (2003) y Moterle et al. (2011) en maíz. Por otro lado, un mayor valor de los cuadrados medios de los efectos de ACG que los de ACE en ambos grupos de características (Cuadros 2 y 3), indica que los efectos aditivos son más importantes que los dominantes en la calidad de semilla y vigor de plántula (Baker, 1978). Otros investigadores (Cervantes-Ortiz et al., 2007; Esquivel et al., 2009; Revilla et al., 1999) han confirmado la importancia de los efectos genéticos aditivos en el vigor de plántula; mientras que Moreno-González (1988) no encontró significancia estadística para ACE en el vigor inicial de plántula, lo mismo que Moterle et al. (2011) para velocidad de emergencia e índice de vigor evaluado en cama de arena.

Los resultados de esta investigación también muestran efecto estadístico significativo para ACE en la mayoría de los rasgos medidos en el grupo de líneas probadas. Estos resultados son similares a los de Cervantes et al. (2006), quienes afirmaron que los efectos de ACE fueron los de mayor importancia para vigor de semilla desarrollada con la prueba de envejecimiento acelerado. En este sentido, se establece que cuando en una población los efectos de acción génica aditiva son más importantes que los efectos de acción génica no aditiva, se recomienda mejorar la población por selección recurrente; por el contrario, si los efectos de acción génica no aditiva son los más importantes, la población debe mejorarse por hibridación, tal como ha sido reportado por otros autores (De la Cruz-Lázaro et al., 2010; Escorcia-Gutiérrez et al., 2010; Esquivel et al., 2009).

Los efectos recíprocos fueron significativos $(P \leq 0.01)$, indicativo de que las características de calidad de semilla y vigor de plántula son afectados por el progenitor femenino utilizado en la producción de semilla. Estos resulta- dos coinciden con los obtenidos por Revilla et al. (1999) y Cervantes et al. (2006) en maíz, quienes señalaron la importancia del progenitor femenino en la calidad de la semilla. También De la Torre y Biasutti (2015) resaltaron la importancia de la selección parental en laboratorio al igual que en etapas tempranas en campo. Del mismo modo, Cervantes-Ortiz et al. (2007) observaron que los efectos recíprocos del vigor inicial de plántula se relacionaron con los de porcentaje de emergencia, índice de vigor y altura de plántula.

Esto sugiere que las líneas endogámicas con buen vigor inicial de plántula tienen potencial para usarse como progenitores femeninos en la producción de semilla híbrida y generar semilla vigorosa. Los bajos coeficientes de variación del análisis estadístico de las características evaluadas confirman la alta confiabilidad de los resultados obtenidos. De acuerdo con los estimadores de los efectos de ACG, las líneas TNM-5 y TNM-4 fueron consistentes con los valores positivos más altos (Cuadro 4). En contraste, la línea TNM-2 presentó el peor comportamiento con estimadores negativos para todas las características de calidad de semilla.

Para características de vigor inicial de plántula, las líneas TNM-5 y TNM-4 sobresalen con efectos altos de ACG y, de igual manera, la línea TNM-2 fue consistente con valores negativos en las características evaluadas. La línea TNM5 tuvo efectos altos de ACG en cada prueba, por lo que podría contribuir al desarrollo de vigor de la semilla y de la plántula (Cuadro 5). Con estos resultados se avanzaría en el nivel de endogamia de estas líneas $\mathrm{S}_{3}$ (TNM-5 y TNM-4) porque fueron las que presentaron mayores efectos aditivos, para posteriormente usarlas en la formación de híbridos para la región de El Bajío.

En los efectos de ACE, las cruzas TNM- $1 \times$ TNM-2, TNM$1 \times$ TNM-3 y TNM-5 $\times$ TNM- 1 presentaron los mayores efectos significativos en biomasa de semilla (BS). En la velocidad de germinación (VG) al primer conteo, sobresalieron las cruzas TNM-3 $\times$ TNM-4, TNM- $1 \times$ TNM-2, TNM-1 $\times$ TNM-5 y TNM-2 $\times$ TNM-4 con efectos altos y positivos. En germinación estándar (GE) fueron eficientes las cruzas TNM-1 $\times$ TNM-2, TNM-2 $\times$ TNM-5 y TNM-3 $\times$ TNM-2, al ser consistentes con los efectos más altos. Finalmente, en el vigor de semilla a través de la prueba de envejecimiento acelerado (EA), las cruzas TNM- $1 \times$ TNM $-2, T N M-2 \times$ TNM- 5 y TNM- $5 \times$ TNM-2 presentaron valores altos y positivos (Cuadros 6 y 7 ).

Los efectos de los estimadores para vigor de plántula (Cuadros 8 y 9) mostraron que en emergencia (E) las cruzas TNM-1 × TNM-2, TNM-3 × TNM-4 y TNM-2 × TNM-5 presentaron valores altos y positivos, para altura de plántula 
Cuadro 4. Efectos de la aptitud combinatoria general (ACG) para características de calidad física y fisiológica de semilla en cinco líneas $\mathrm{S}_{3}$ de maíz.

\begin{tabular}{|c|c|c|c|c|}
\hline Línea & BS (g) & VG (\%) & GE (\%) & EA (\%) \\
\hline TNM-1 & $0.7 * \star$ & -1.0 & $3.8^{* *}$ & 0.6 \\
\hline TNM-2 & $-3.2 * \star$ & $-6.8 * *$ & $-7.7 \star \star$ & $-14.6 * *$ \\
\hline TNM-3 & $-1.1 * \star$ & $-5.6 * \star$ & $-1.3 *$ & 0.0 \\
\hline TNM-4 & -0.2 & $5.3 * \star$ & 0.4 & $3.7 * \star$ \\
\hline TNM-5 & $3.8 * \star$ & 8.1 ** & $4.7 \star \star$ & $10.2 * \star$ \\
\hline
\end{tabular}

$\star, * *$ Diferente de cero a una probabilidad de 0.05 y 0.01 , respectivamente. BS: biomasa de semilla; VG: velocidad de germinación (4º día); GE: germinación estándar; EA: vigor a través de la prueba de envejecimiento acelerado.
Cuadro 5. Efectos de la aptitud combinatoria general (ACG) para características de vigor inicial de plántula en líneas $\mathrm{S}_{3}$ de maíz.

\begin{tabular}{|c|c|c|c|c|c|}
\hline Línea & $E(\%)$ & APL $(\mathrm{cm})$ & BSV (g) & IVI-I & IV-II \\
\hline TNM-1 & $2.22 * \star$ & 0.03 & -0.01 & 17.1 & $-8.8 * \star$ \\
\hline TNM-2 & $-8.58 * \star$ & $-1.58 * \star$ & $-0.23 * *$ & $-211.5 * \star$ & $-39.1 * *$ \\
\hline TNM-3 & $-3.08 * *$ & $0.31 * *$ & 0.05 & -2.9 & $5.7 *$ \\
\hline TNM-4 & $3.82 * *$ & 0.07 & 0.06 & $40.9 * \star$ & $8.6 * *$ \\
\hline TNM-5 & $5.62 * *$ & $1.15 \star *$ & $0.13 *$ & $156.3 * *$ & $33.7 * *$ \\
\hline
\end{tabular}

*,** Diferente de cero a una probabilidad de 0.05 y 0.01 , respectivamente E: emergencia; APL: altura de plántula; BSV: biomasa seca de vástago; IV-I: índice de vigor I; IV-II: índice de vigor II.

Cuadro 6. Efectos de la aptitud combinatoria específica (ACE) de 10 cruzas directas de maíz para características de calidad física y fisiológica de semilla.

\begin{tabular}{|c|c|c|c|c|}
\hline Cruzas & $\mathrm{BS}(\mathrm{g})$ & VG (\%) & GE $(\%)$ & EA (\%) \\
\hline TNM-1 $\times$ TNM-2 & $3.32 * *$ & $19.10 * \star$ & $7.18 * *$ & $23.16 * *$ \\
\hline TNM-1 $\times$ TNM-3 & $3.41 * *$ & 3.24 & -0.54 & $8.49 * *$ \\
\hline TNM- $1 \times$ TNM-4 & -0.21 & $5.57 \star$ & 0.98 & $5.49 * \star$ \\
\hline TNM-1 $\times$ TNM-5 & 0.16 & $8.10 *$ & $-1.94 \star$ & 0.96 \\
\hline TNM-2 $\times$ TNM-3 & $-0.71 *$ & $-12.62 * *$ & $-13.34 * *$ & $-8.57 * *$ \\
\hline TNM-2 $\times$ TNM-4 & $1.32 * *$ & $6.04 *$ & $-5.48 * *$ & $3.42 *$ \\
\hline TNM-2 $\times$ TNM-5 & $0.98 *$ & 1.24 & $4.92 * *$ & $18.49 * *$ \\
\hline TNM-3 $\times$ TNM-4 & $-2.20 * *$ & $26.17 * *$ & -0.66 & $8.09 * *$ \\
\hline TNM-3 $\times$ TNM-5 & $-1.45 * *$ & -3.29 & 0.66 & 0.89 \\
\hline TNM-4 × TNM-5 & 0.17 & 1.70 & -0.66 & $-6.10 * *$ \\
\hline
\end{tabular}

*,** Diferente de cero a una probabilidad de 0.05 y 0.01 , respectivamente. BS: biomasa de semilla; VG: velocidad de germinación (4 día); GE: germinación estándar; EA: vigor a través de la prueba de envejecimiento acelerado.

Cuadro 7. Efectos de la aptitud combinatoria específica (ACE) de 10 cruzas recíprocas de maíz para características de calidad física y fisiológica de semilla.

\begin{tabular}{|c|c|c|c|c|}
\hline Cruzas & BS (g) & VG (\%) & GE (\%) & EA (\%) \\
\hline TNM-2 $\times$ TNM-1 & $4.70 * *$ & 1.33 & 1.33 & -3.33 \\
\hline TNM-3 $\times$ TNM-1 & -0.55 & 4.00 & $2.66 *$ & -2.00 \\
\hline TNM-4 × TNM- 1 & $1.37 * *$ & 4.00 & 0.66 & -4.00 \\
\hline TNM-5 $\times$ TNM-1 & $2.38 * \star$ & $-6.66 *$ & 0.66 & -0.66 \\
\hline TNM-3 $\times$ TNM-2 & $1.71 \star \star$ & $-7.66 *$ & $20.33 * *$ & -0.66 \\
\hline TNM-4 × TNM-2 & $-1.46 * *$ & $-12.00 * *$ & $-20.00 * \star$ & $-18.66 * *$ \\
\hline TNM-5 $\times$ TNM-2 & $-1.10 *$ & 2.00 & $-2.66^{*}$ & $14.89 * \star$ \\
\hline TNM-4 × TNM-3 & $2.02 \star \star$ & $-6.66 * \star$ & -0.66 & 0.66 \\
\hline TNM-5 $\times$ TNM-3 & $-1.24 \star$ & 2.66 & 0.66 & -2.66 \\
\hline TNM-5 $\times$ TNM-4 & -0.65 & 0.00 & -0.66 & -3.33 \\
\hline
\end{tabular}

*,** Diferente de cero a una probabilidad de 0.05 y 0.01 , respectivamente. BS: biomasa de semilla; VG: velocidad de germinación (40 día); GE: germinación estándar; EA: vigor a través de la prueba de envejecimiento acelerado. 
(APL) las cruzas TNM- $1 \times$ TNM-3, TNM- $5 \times$ TNM- 1 y TNM$3 \times \mathrm{TNM}-4$ fueron consistentes con los estimadores más altos; mientras que, en biomasa seca de vástago (BSV) lo hicieron las cruzas entre las líneas TNM-5 × TNM-1, TNM$4 \times$ TNM-2 y TNM-1 × TNM-3; para el índice de vigor I y II sobresalen las cruzas TNM-3 $\times$ TNM-4, TNM-5 $\times$ TNM-1, TNM-1 $\times$ TNM-3, TNM-3 $\times$ TNM-1 y TNM- $2 \times$ TNM -5 con efectos altos y positivos.

De acuerdo con los resultados anteriores, no hubo consistencia entre las líneas con la mejor ACG y las combinaciones para ACE. Al respecto, Revilla et al. (1999) destacan la ventaja de un mayor avance genético en maíz cuando se desarrolla una selección de combinaciones de híbridos específicos para mejorar vigor de plántula en maíz, que al optar sólo con base en efectos provocados por la ACG de las líneas.
Las líneas TNM-4 y TNM-5 mostraron efectos positivos de ACG para la mayoría de las características evaluadas y al combinarse entre ellas o con las demás líneas mostraron valores superiores de ACE (Cuadros 4 y 5), mientras que la línea TNM-2, consistentemente tuvo valores negativos de ACG. Sin embargo, en varias cruzas hubo efectos de ACE positivos y significativos en las características descritas anteriormente. Estos resultados coincidieron con los obtenidos por Reyes et al. (2004) y Cervantes et al. (2006), quienes señalaron que la cruza simple tendrá valor alto de ACE si al menos una de sus líneas muestra alta ACG, aunque también se observaron fuertes diferencias entre las cruzas para las características estudiadas, por lo que indica que éstos no pueden ser predichos con los valores de ACG de los progenitores (Baker, 1978).

En biomasa de semilla (BS), la heterosis varió de -2.7 a

Cuadro 8. Efectos de la aptitud combinatoria específica (ACE) de 10 cruzas directas de maíz para características de vigor inicial de plántula.

\begin{tabular}{|c|c|c|c|c|c|}
\hline Cruza & $\mathrm{E}(\%)$ & $\mathrm{APL}(\mathrm{cm})$ & BSV (g) & IV-I & IV-II \\
\hline TNM-1 × TNM-2 & $13.28 * \star$ & -0.07 & 0.04 & $106.78 * *$ & $24.35 * *$ \\
\hline TNM-1 × TNM-3 & -1.72 & $1.39 * *$ & $0.29^{*}$ & $93.64 * *$ & 34.61 ** \\
\hline TNM-1 × TNM-4 & $-5.12 \star \star$ & $0.46 * \star$ & -0.03 & -8.32 & -0.67 \\
\hline TNM-1 × TNM-5 & 1.08 & 0.19 & 0.04 & $44.92 \star$ & -0.75 \\
\hline TNM-2 × TNM-3 & 1.08 & -0.04 & 0.20 & -1.90 & 4.59 \\
\hline TNM-2 × TNM-4 & -1.32 & $-0.44 * *$ & -0.06 & $-61.81 * \star$ & $-12.49 *$ \\
\hline TNM-2 $\times$ TNM-5 & $3.88 *$ & $0.82 \star \star$ & -0.02 & $88.96 * \star$ & $21.69 \star \star$ \\
\hline TNM-3 $\times$ TNM-4 & $7.18^{* *}$ & $1.18 \star \star$ & 0.04 & $184.47 * \star$ & $38.82 * *$ \\
\hline TNM-3 × TNM-5 & 1.38 & $0.50 * *$ & -0.10 & $57.55 * *$ & -6.96 \\
\hline TNM-4 × TNM-5 & -3.02 & $0.37 *$ & -0.03 & 9.03 & -2.96 \\
\hline
\end{tabular}

*, ** Diferente de cero a una probabilidad de 0.05 y 0.01, respectivamente. E: emergencia; APL: altura de plántula; BSV: biomasa seca de vástago; IV-I: índice de vigor I; IV-II: índice de vigor II.

Cuadro 9. Efectos de la aptitud combinatoria específica (ACE) de 10 cruzas recíprocas de maíz para características de vigor inicial de plántula.

\begin{tabular}{|c|c|c|c|c|c|}
\hline Cruza & $E(\%)$ & $\mathrm{APL}(\mathrm{cm})$ & $\mathrm{BSV}(\mathrm{g})$ & IV-I & IV-II \\
\hline TNM-2 2 TNM-1 & -1.00 & -0.12 & -0.062 & 20.55 & -3.74 \\
\hline TNM-3 $\times$ TNM-1 & $-10.50 * *$ & 0.07 & 0.047 & $123.99 * \star$ & $37.54 \star \star$ \\
\hline TNM-4 × TNM-1 & 1.00 & -0.30 & -0.312 & -14.84 & $-34.78 * *$ \\
\hline TNM-5 $\times$ TNM-1 & 3.00 & $1.14 * *$ & $0.315 * \star$ & $145.65 \star \star$ & $30.18 * \star$ \\
\hline TNM-3 $\times$ TNM-2 & 3.50 & $-0.60 * *$ & -0.105 & -11.77 & -1.98 \\
\hline TNM-4 × TNM-2 & $-4.00 *$ & $0.54 * *$ & $0.310 *$ & 8.88 & $19.10 * *$ \\
\hline TNM-5 × TNM-2 & 3.00 & -0.31 & -0.107 & 5.86 & 9.88 \\
\hline TNM-4 $\times$ TNM-3 & 0.00 & $0.85 * \star$ & -0.002 & $79.35 * \star$ & -8.82 \\
\hline TNM-5 $\times$ TNM-3 & 2.00 & $0.80 * *$ & 0.085 & $98.07 * *$ & $30.26 * *$ \\
\hline TNM- $5 \times$ TNM-4 & -1.50 & 0.31 & -0.030 & 10.90 & -10.53 \\
\hline
\end{tabular}

*,** Diferente de cero a una probabilidad de 0.05 y 0.01, respectivamente. E: emergencia; APL: altura de plántula; BSV: biomasa seca de vástago; IV-I: índice de vigor I; IV-II: índice de vigor II. 
$48.9 \%$. Del total de las cruzas, 19 presentaron heterosis positiva, donde sobresalen las cruzas TNM-1 $\times$ TNM-2, TNM-3 $\times$ TNM-1, TNM-3 $\times$ TNM-4 y TNM-4 $\times$ TNM-2 con valores de heterosis superiores al $20 \%$. Para velocidad de germinación (VG), 19 de las 20 cruzas presentaron heterosis positiva y sobresalieron las cruzas TNM-1 $\times$ TNM-2 y TNM-3 $\times$ TNM-4 con heterosis superior de $100 \%$. En germinación estándar (GE), la heterosis varió de -44 a 15.3\%.

Finalmente, en el vigor de la semilla medido a través de la prueba de envejecimiento acelerado (EA), sobresalieron 13 cruzas con heterosis superior a $20 \%$ y de estas, tres cruzas presentaron heterosis mayores a $50 \%($ TNM-1 $\times$ TNM-2, TNM-2 × TNM-5 y TNM-5 × TNM-2) (Cuadros 10 y 11). La heterosis es el fenómeno en el que la $F_{1}$, resul- tante del cruzamiento entre dos genotipos, es superior a los progenitores en crecimiento, tamaño, rendimiento y vigor (Gutiérrez et al., 2002). En este contexto, Gutiérrez et al. (2002) y De la Cruz et al. (2003) mencionaron que en el mejoramiento genético de maíz el nivel deseable para aprovechamiento de la heterosis en una cruza es cuando menos de $20 \%$.

Para características de vigor inicial de plántula (Cuadros 12 y 13); en el porcentaje de emergencia (E), la heterosis varió de -14.8 a 33 \%. Por otro lado, 15 de las 20 cruzas presentaron heterosis positiva. En altura de plántula (APL), las cruzas TNM-1 $\times$ TNM-3, TNM-3 $\times$ TNM-4 y TNM-5 $\times$ TNM-1 las heterosis fueron de 29.3, 17.9, 15.2 y $15.1 \%$, respectivamente. Para biomasa seca de vástago (BSV), se

Cuadro 10. Heterosis porcentual con respecto al progenitor medio de 10 cruzas directas de maíz para características de calidad de semilla.

\begin{tabular}{lcccc}
\hline Cruzas & BS $(\mathrm{g})$ & VG $(\%)$ & GE $(\%)$ & EA (\%) \\
\hline TNM-1 × TNM-2 & 48.9 & 130.0 & 8.5 & 150.9 \\
TNM-1 × TNM-3 & 25.5 & 60.0 & 3.1 & 47.3 \\
TNM-1 × TNM-4 & 19.5 & 49.2 & 7.2 & 39.4 \\
TNM-1 × TNM-5 & 15.3 & 74.3 & 4.8 & 33.9 \\
TNM-2 $\times$ TNM-3 & 18.6 & -16.1 & 1.4 & 40.0 \\
TNM-2 $\times$ TNM-4 & 14.1 & 72.5 & -30.7 & 12.5 \\
TNM-2 TNM-5 & 6.5 & 24.1 & 2.2 & 60.0 \\
TNM-3 TNM-4 & 24.3 & 113.1 & 7.2 & 23.3 \\
TNM-3 × TNM-5 & -2.7 & 16.8 & 3.4 & 9.2 \\
TNM-4 × TNM-5 & 3.1 & 49.1 & 6.4 & 1.1 \\
\hline
\end{tabular}

BS: biomasa de semilla; VG: velocidad de germinación ( $4^{\circ}$ día); GE: germinación estándar; EA: vigor a través de la prueba de envejecimiento acelerado.

Cuadro 11. Heterosis porcentual con respecto al progenitor medio de 10 cruzas recíprocas de maíz para características de calidad de semilla.

\begin{tabular}{lcccc}
\hline Cruzas & BS $(\mathrm{g})$ & VG $(\%)$ & GE $(\%)$ & EA (\%) \\
\hline TNM-2 $\times$ TNM-1 & 15.7 & 52.1 & 5.9 & 26.1 \\
TNM-3 $\times$ TNM-1 & 13.9 & 56.7 & -2.8 & 43.6 \\
TNM-4 $\times$ TNM-1 & 10.6 & 55.7 & 5.8 & 10.3 \\
TNM-5 $\times$ TNM-1 & 20.8 & 59.6 & 3.4 & 31.6 \\
TNM-3 $\times$ TNM-2 & 6.5 & 20.9 & -44.0 & -1.3 \\
TNM-4 $\times$ TNM-2 & 18.3 & 53.5 & 15.3 & 42.5 \\
TNM-5 $\times$ TNM-2 & 11.3 & 17.2 & 8.1 & 62.2 \\
TNM-4 $\times$ TNM-3 & 11.5 & 55.9 & 8.7 & 21.6 \\
TNM-5 $\times$ TNM-3 & 3.8 & 13.6 & 2.0 & 15.3 \\
TNM-5 $\times$ TNM-4 & 6.5 & 43.0 & 7.9 & 8.9 \\
\hline BS TNM
\end{tabular}

BS: biomasa de semilla; VG: velocidad de germinación (4 día); GE: germinación estándar; EA: vigor a través de la prueba de envejecimiento acelerado. 
encontraron 13 cruzas con efectos de heterosis positivos y nueve de estas cruzas superan valores de $20 \%$ en heterosis.

En índice de vigor I (IV-I), las cruzas con los valores más altos de heterosis fueron TNM-2 $\times$ TNM-1, TNM-2 $\times$ TNM5, TNM-1 × TNM-5 y TNM- $1 \times$ TNM-2. Finalmente, para el índice de vigor II (IV-II), las mejores cruzas fueron TNM-5 $\times$ TNM-2, TNM-3 $\times$ TNM-1, TNM-2 $\times$ TNM-1, TNM-3 $\times$ TNM2, TNM-2 $\times$ TNM-3, TNM-1 $\times$ TNM-2, TNM-1 $\times$ TNM-3 y TNM-5 $\times$ TNM-1.

Estos resultados coinciden parcialmente con los reportados por Esquivel et al. (2009) quienes realizaron un estudio de heterosis en poblaciones de maíz en etapas tempranas de desarrollo y encontraron valores de heterosis de hasta $41.8 \%$ en velocidad de emergencia, $27.6 \%$ para porcentaje de emergencia, $14.8 \%$ en altura de plántula, $15.6 \%$ para longitud de mesocotilo y $31.1 \%$ en biomasa total, lo que atribuyeron a la divergencia genética por distinto origen geográfico entre las poblaciones. En este sentido, Romero et al. (2002) mencionaron que la expresión de la heterosis es un indicador de la divergencia genética, aunque la ausencia de ella no necesariamente infiere falta de divergencia. En todas las variables registradas se presentaron valores de heterosis negativos, lo que indica que no existe heterosis y esta condición puede deberse a que existen alelos comunes para estas características (De la Cruz-Lázaro et al., 2010).

Con base en los resultados expuestos, se puede inferir que los valores de heterosis dependen del valor y signo del efecto de ACE (sij); es decir, valores positivos altos de heterosis corresponden a efectos sij positivos altos y viceversa,

Cuadro 12. Heterosis porcentual con respecto al progenitor medio de 10 cruzas directas de maíz para características de vigor inicial de plántula.

\begin{tabular}{|c|c|c|c|c|c|}
\hline Cruza & $E(\%)$ & $\mathrm{APL}(\mathrm{cm})$ & BSV (g) & IV-I & IV-II \\
\hline TNM-1 × TNM-2 & 27.7 & 2.2 & 21.3 & 26.5 & 48.6 \\
\hline TNM-1 × TNM-3 & -6.0 & 29.3 & 57.6 & 17.9 & 48.1 \\
\hline TNM-1 × TNM-4 & -3.2 & -1.4 & -12.8 & -6.5 & -19.1 \\
\hline TNM-1 × TNM-5 & 10.1 & 14.9 & 30.4 & 27.2 & 42.5 \\
\hline TNM-2 $\times$ TNM-3 & 15.9 & 1.9 & 33.8 & 15.9 & 49.9 \\
\hline TNM-2 × TNM-4 & 1.2 & -1.8 & 20.1 & -3.8 & 9.4 \\
\hline TNM-2 × TNM-5 & 20.0 & 9.5 & -6.5 & 27.7 & 9.0 \\
\hline TNM-3 × TNM-4 & 20.8 & 15.1 & 15.0 & 16.2 & 16.8 \\
\hline TNM-3 × TNM-5 & 10.4 & 13.1 & 9.5 & 20.2 & 15.7 \\
\hline TNM-4 × TNM-5 & -14.8 & -13.6 & -5.6 & -30.0 & -19.2 \\
\hline
\end{tabular}

E: emergencia; APL: altura de plántula; BSV: biomasa seca de vástago; IV-I: índice de vigor I; IV-II: índice de vigor II.

Cuadro 13. Heterosis porcentual con respecto al progenitor medio de 10 cruzas recíprocas de maíz para características de vigor inicial de plántula.

\begin{tabular}{lccccc}
\hline Cruza & $\mathrm{E}(\%)$ & APL $(\mathrm{cm})$ & BSV $(\mathrm{g})$ & IV-I & IV-II \\
\hline TNM-2 × TNM-1 & 13.3 & 8.1 & 31.6 & 36.7 & 65.3 \\
TNM-3 × TNM-1 & 13.0 & 17.5 & 50.0 & 31.1 & 70.1 \\
TNM-4 × TNM-1 & -5.4 & 8.4 & 27.5 & 0.60 & 14.8 \\
TNM-5 × TNM-1 & 3.3 & 15.2 & -7.9 & 16.8 & 28.0 \\
TNM-3 × TNM-2 & 4.3 & 1.8 & 51.6 & 18.4 & 52.7 \\
TNM-4 × TNM-2 & 19.4 & -9.4 & 21.8 & 3.4 & -16.9 \\
TNM-5 × TNM-2 & 12.0 & 7.9 & 7.2 & 17.6 & 75.8 \\
TNM-4 × TNM-3 & 6.8 & 7.5 & 15.0 & 13.0 & 19.0 \\
TNM-5 × TNM-3 & 2.3 & 7.9 & -0.5 & 11.8 & 0.1 \\
TNM-5 × TNM-4 & -4.2 & 3.1 & -0.5 & -1.7 & -8.6 \\
\hline
\end{tabular}

E: emergencia; APL: altura de plántula; BSV: biomasa seca de vástago; IV-I: índice de vigor I; IV-II: índice de vigor II. 
y que los valores bajos de heterosis corresponden a efectos sij positivos o negativos bajos, tal como fue reportado por Escorcia-Gutiérrez et al. (2010).

\section{CONCLUSIONES}

Los efectos de aptitud combinatoria general fueron el principal componente en todas las características evaluadas; también se detectaron efectos estadísticos de aptitud combinatoria específica y efectos recíprocos en este grupo de genotipos.

Se presentaron efectos recíprocos significativos para todas las características, lo que indica la importancia de seleccionar apropiadamente el progenitor femenino para favorecer la calidad de la semilla y el vigor de la plántula. Se demostró que la heterosis se manifiesta en el vigor de la semilla y desde estados tempranos de desarrollo de la plántula de maíz.

Las líneas TNM-4 y TNM-5 mostraron alta ACG como progenitores y alta ACE en combinaciones híbridas superiores; por lo que estas líneas podrían ser mejoradas por algún método de selección recurrente para incrementar la frecuencia genética aditiva en la calidad de la semilla y vigor de plántula y posteriormente formar híbridos con mejor calidad de semilla.

\section{BIBLIOGRAFÍA}

Allard R. W. (1960) Principles of Plant Breeding. John Wiley and Sons, Inc. New York. London. $485 \mathrm{p}$

Antuna G. O., F. Rincón S., E. Gutiérrez R., N. A. Ruiz T. y L. Bustamante G. (2003) Componentes genéticos de caracteres agronómicos y de calidad fisiológica de semillas en líneas de maíz. Revista Fitotecnia Mexicana 26:11-17.

Baker R. J. (1978) Issues in diallel analysis. Crop Science 18:533-536.

Barla-Szabo G., J. Bocsi, B. Dolinka and M. Odiemah (1989) Diallel analysis of seed vigour in maize. Seed Science and Technology 18:721729.

Cai Q. S., L. L. Wang, W. H. Yao, Y. D. Zhang, L. Liu, L. J. Yu and X. M. Fan (2012) Diallel analysis of photosynthetic traits in maize. Crop Science 52:551-559.

Cervantes 0. F., G. García-De Los Santos, A. Carballo-Carballo, D. Bergvinson, J. L. Crossa, E. Mendoza-Elos y E. Moreno-Martínez (2006) Análisis dialélico para caracteres de vigor de semilla y de plántula en genotipos de maíz tropical. Agricultura Técnica en México 32:77-87.

Cervantes-Ortiz F., G. García-De Los Santos, A. Carballo-Carballo, D. Bergvinson, J. L. Crossa, M. Mendoza-Elos y E. Moreno-Martínez (2007) Herencia del vigor de plántula y su correlación con caracteres de planta adulta en líneas endogámicas de maíz tropical. Agrociencia 41:425-433.

Crow J. F. (1999) Dominance and overdominance. In: Genetics and Explotation of Heterosis in Crops. J. G. Coors and S. Pandey (eds.). American Society of Agronomy, Inc. Crop Science Society of America, Inc. Soil Science Society of America, Inc. Madison, Wisconsin, USA. pp: 49-58.

De la Cruz L. E., E. Gutiérrez R., A. Palomo G. y S. Rodríguez H. (2003) Aptitud combinatoria y heterosis de líneas de maíz en la Comarca Lagunera. Revista Fitotecnia Mexicana 26:279-284.

De la Cruz-Lázaro E., G. Castañón-Nájera, N. P. Brito-Manzano, A. GómezVázquez, V. Robledo-Torres y A. J. Lozano R. (2010) Heterosis y apti- tud combinatoria de poblaciones de maíz tropical. International Journal of Experimental Batany 79:11-17.

De la Torre M. V. y C. A. Biasutti (2015) Efectos recíprocos y aptitud combinatoria en caracteres relacionados al vigor temprano y al rendimiento en grano en maíz. Agriscientia 32:41-53.

Escorcia-Gutiérrez N., J. D. Molina-Galán, F. Castillo-González y A. MejíaContreras (2010) Rendimiento, heterosis y depresión endogámica de cruzas simples de maíz. Revista Fitotecnia Mexicana 33:271-279.

Esquivel E. G., F. Castillo G., J. M. Hernández C., A. Santacruz V., G. García S., J. Acosta G. y A. Ramírez H. (2009) Aptitud combinatoria y heterosis en etapas tempranas del desarrollo del maíz. Revista Fitotecnia Mexicana 32:311-318.

Falconer D. S. and T. F. C. Mackay (1996) Introduction to Quantitative Genetics. 4th ed. Longman. Essex, England. 464 p.

Finch-Savage W. E. (1994) Influence of seed quality on crop establishment, growth and yield. In: Seed Quality: Basic Mechanisms and Agricultural Implications. A. S. Basra (ed.). Food Products Press. New York. pp: 361-384.

Goggi A. S., P. Caragea, L. Pollak, G. McAndrews, M. DeVries and K. Montgomery (2008) Seed quality assurance in maize breeding programs: tests to explain variations in maize inbreeds and populations Agronomy Journal 100:337-343.

Gomes M. S., E. V. R. Von Pinho, R. G. Von Pinho and M. G. G. C. Vieira (2000) Estimativas da capacidade de combinação de linhagens de milho tropical para qualidade fisiológica de sementes. Ciência e Agrotecnologia, Lavras 24 (Edicão Especial):41-49.

Griffing B. (1956) Concept of general and specific combining ability in relation to diallel crossing systems. Australiam journal of Biological Sciences 9:463-493.

Guillen-De la Cruz P., E. de la Cruz-Lázaro, G. Castañón-Nájera, R. OsorioOsorio, N. P. Brito-Manzano, A. Lozano-Del Río y U. López-Noverola (2009) Aptitud combinatoria general y específica de germoplas ma tropical de maíz. Tropical and Subtropical Agroecosystems 10:101-107.

Gutiérrez R. E., A. Palomo G., A. Espinosa B. y E. De la Cruz L. (2002) Aptitud combinatoria y heterosis para rendimiento de líneas de maíz en la Comarca Lagunera, México. Revista Fitotecnia Mexicana 25:271-277.

Hallauer A. R. and J. B. Miranda (1981) Quantitative Genetics in Maize Breeding. lowa State University Press. Ames, lowa, USA. 468 p.

Hoecker N., B. Keller, H. P. Piepho and F. Hochholdinger (2006) Manifestation of heterosis during early maize (Zea mays L.) root development. Theoretical and Applied Genetics 112:421-429.

ISTA, International Seed Testing Association (1995) Handbook of Vigor Test Methods. 3rd ed. International Seed Testing Association. Zürich. 117 p.

Marcos-Filho J. M. (2005) Fisiologia de Sementes de Plantas Cultivadas. Associacão Brasileira de Tecnologia de Sementes. Piracicaba Brasil. $546 p$

Melani M. D. and M. J. Carena (2005) Alternative maize heterotic patterns for the Northern corn belt. Crop Science 45:2186-2194.

Moreno-González J. (1988) Diallel crossing system in sets of flint and dent inbred lines of maize (Zea mays L.). Maydica 33:37-49.

Moterle L. M., A. L. Braccini, C. A. Scapim, R. J. B. Pinto, L. S. A. Goncalves, R. Rordrigues and A. T. Do Amaral Jr. (2012) Combining ability of popcorn lines for seed quality and agronomic traits. Euphytica 185:337-347.

Moterle L. M., A. L. Braccini, C. A. Scapim, R. J. B. Pinto, L. S. A. Goncalves, A. T. Do Amaral Jr. and T. R. C. Silva (2011) Combining ability of tropical maize lines for seed quality and agronomic traits. Genetics and Molecular Research 10:2268-2278.

Munamava M. R., A. S. Goggi and L. Pollak (2004) Seed quality of maize inbred lines with different composition and genetic backgrounds. Crop Science 44:542-548.

Popinigis F. (1985) Fisiologia da Semente. 2nd ed. Agiplan. Brasilia. 289 p.

Revilla P., A. Butrón, R. A. Malval and R. A. Ordás (1999) Relationships among kernel weight, early vigor and growth in maize. Crop Science 39:654-658.

Reyes L. D., J. D. Molina G., M. A. Oropeza R. y E. C. Moreno P. (2004) Cruzas dialélicas entre líneas autofecundadas de maíz derivadas de la raza Tuxpeño. Revista Fitotecnia Mexicana 27:49-56.

Romero P. J., F. Castillo G. y R. Ortega P. (2002) Cruzas de poblaciones nativas de maíz de la raza Chalqueno: Il. Grupos genéticos, 
divergencia genética y heterosis. Revista Fitotecnia Mexicana 25:107-115

Silva C. P. D., A. Teixeira A. Jr., H. Duarte V., J. Saltires S., I. L. J. Freitas and M. Gonzaga P. (2013) Genetic effects on seed quality in diallel crosses of popcorn. Ciência e Agrotecnologia, Lavras 37:502-511.

TeKrony D. M. and D. B. Egli (1991) Relationship of seed vigor to crop yield: a review. Crop Science 31:816-822.

Vergara A. N., S. A. Rodríguez H. y H. S. Córdova 0. (2005) Aptitud combinatoria general y específica de líneas de maíz (Zea mays) tropical y subtropical. Agronomía Mesoamericana 16:137-143.

Yan W. and M. S. Kang (2003) GGE Biplot Analysis. A Graphical Tool for Breeders, Geneticists and Agronomists. CRC Press. New York. $228 p$.

Zhang Y. and M. S. Kang (2003) Diallel-SAS: a program for Griffing's diallel methods. In: Handbook of Formulas and Software for Plant Geneticists and Breeders. M. S. Kang (ed.). Food Products Press. New York. pp:1-19. 